\title{
Polycyclic Aromatic Hydrocarbon with Embedded Azulene Unit
}

Embedding of azulene structure and electronics into a PAH:
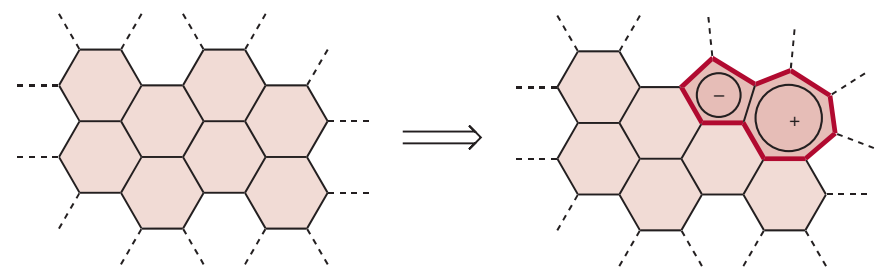

Synthesis of azulenium- and azulene-embedded PAH:

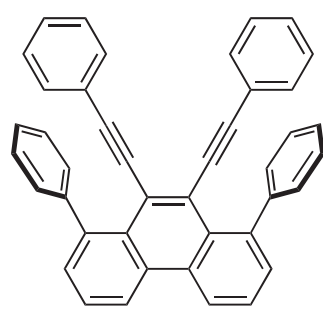

$\mathrm{AgBF}_{4}$

chlorobenzene

$1 \mathrm{~d}$

$-\mathrm{Ag}$
$78 \%$ yield

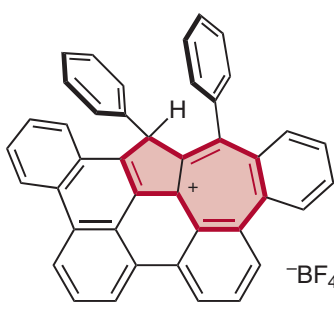

$2 \mathrm{H}^{+}$

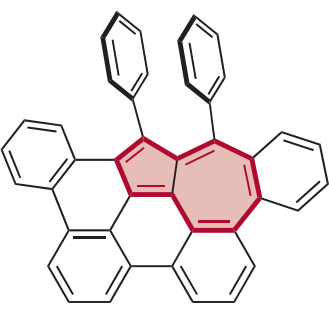

2
Significance: Incorporation of heptagons and pentagons into a polycyclic aromatic hydrocarbon (PAH) core leads to changes in the molecular geometry and electronic properties of the original $\mathrm{PAH}$. This report demonstrates the use of silver(I) cations to achieve an efficient synthesis of an azulene-embedded PAH 2, which is a challenging issue that is plagued by limited methodologies.
Comment: Two-electron oxidation of the silver(l) ion promoted a three-fold transannulation reaction between the aromatic and acetylene units of $\mathbf{1}$ resulting in the selectively embedded azulenium cation $\mathbf{2 H}^{+}$, which could be subsequently deprotonated by trimethylamine to afford the neutral azuleneembedded PAH 2. Compound 2 exhibited a lowenergy absorption band featuring characteristics of the non-alternant moiety, along with reversible one-electron redox events for the oxidation or reduction of $\mathbf{2}$, resulting in tropylium cation or cyclopentadienyl anion scaffolds, respectively. 